\title{
INDUCED HOMOLOGY HOMOMORPHISMS FOR SET-VALUED MAPS
}

\author{
BARRETT O'NEILL
}

$\S 1$. If $X$ and $Y$ are topological spaces, a set-valued function $F$ : $X \rightarrow Y$ assigns to each point $x$ of $X$ a closed nonempty subset $F(x)$ of $Y$. Let $H$ denote Čech homology theory with coefficients in a field. If $X$ and $Y$ are compact metric spaces, we shall define for each such function $F$ a vector space of homomorphisms from $H(X)$ to $H(Y)$ which deserve to be called the induced homomorphisms of $F$. Using this notion we prove two fixed point theorems of the Lefschetz type.

All spaces we deal with are assumed to be compact metric. Thus the group $H(X)$ can be based on a group $C(X)$ of projective chains [4]. Define the support of a coordinate $c_{i}$ of $c \in C(X)$ to be the union of the closures of the kernels of the simplexes appearing in $c_{i}$. Then the intersection of the supports of the coordinates of $c$ is defined to be the support $|c|$ of $c$.

If $F: X \rightarrow Y$ is a set-valued function, let $F^{-1}: Y \rightarrow X$ be the function such that $x \in F^{-1}(y)$ if and only if $y \in F(x)$. Then $F$ is upper (lower) semicontinuous provided $F^{-1}$ is closed (open). If both conditions hold, $F$ is continuous. If $\varepsilon>0$ is a real number, we shall also denote by $\varepsilon: X \rightarrow X$ the set-valued function such that $\varepsilon(x)=\left\{x^{\prime} \mid d\left(x, x^{\prime}\right) \leqq \varepsilon\right\}$ for each $x \in X$.

Let $A$ and $B$ be chain groups with supports in $X$ and $Y$ respectively, and let $\varepsilon>0$ be a number. A chain map $\varphi: A \rightarrow B$ is accurate with respect to a set-valued function $F: X \rightarrow Y$ provided $|\varphi(a)| \subset F(|a|)$ for each $a \in A$. Further, $\varphi$ is $\varepsilon$-accurate with respect to $F$ provided $\varphi$ is accurate with respect to the composite function $\varepsilon F \varepsilon$.

(1) Definition. A homomorphism $h: H(X) \rightarrow H(Y)$ is an induced homomorphism of a set-valued function $F: X \rightarrow Y$ provided that given $\varepsilon>0$ there is a chain map $\varphi: C(X) \rightarrow C(Y)$ such that $\varphi$ is $\varepsilon$-accurate with respect to $F$ and $\varphi_{*}=h$.

We shall say that a homology homomorphism $h$ is nontrivial provided the 0-dimensional component $h_{0}: H_{0}(X) \rightarrow H_{0}(Y)$ is not the zero homomorphism. It will appear that a continuous set-valued function need not have a nontrivial induced homomorphism.

The set of all induced homomorphisms of an arbitrary set-valued function is, under the usual operations, a vector space. If $h_{F}$ and $h_{G}$ are induced homomorphisms of upper semi-continuous functions $F: X \rightarrow Y$ and $G: Y \rightarrow Z$, then $h_{G} h_{F}$ is an induced homomorphism of $G F$. If $F$ :

Received July 9, 1956. 
$X \rightarrow Y$ is a point-valued map of a connected (compact metric) space into a compact polyhedron, then the induced homology homomorphisms of $F$ are exactly the scalar multiples of the Čech homology homomorphism $F_{*}$. Corresponding to each function $F: X \rightarrow Y$, let $F^{\prime}: X \rightarrow X \times Y$ be such that $F^{\prime}(x)=\{x\} \times F(x)$, for $x \in X$. If $h: H(X) \rightarrow H(X \times Y)$ is a nontrivial induced homomorphism of $F^{\prime \prime}$, then $q_{*} h$ is a nontrivial induced homomorphism of $F$, where $q$ is the projection of the productspace on $Y$.

If $T$ is a triangulation of a compact polyhedron $X$, let $C(X, T)$ be the group of oriented simplicial chains based on $T$, with the given field of coefficients. We may assume that the sequence of coverings used to define $C(X)$ consists of the star-coverings associated with the successive barycentric subdivisions of a fixed triangulation of $X$.

(2) Lemma. Let $X$ be a compact polyhedron, $F: X \rightarrow Y$ a set-valued function. Then $h: H(X) \rightarrow H(Y)$ is an induced homomorphism of $F$ if and only if given $\varepsilon>0$ there is an arbitrarily fine triangulation $T$ of $X$ and an e-accurate chain map $\psi: C(X, T) \rightarrow C(Y)$ such that $\psi_{*}=h$.

Proof. Given $\varepsilon>0$, let $T$ be one of the selected triangulations of $X$ with mesh at most $\varepsilon / 2$. Denote by $p$ the projection of $C(X)$ onto the chains of the nerve of the star-covering associated with $T$, followed by the natural isomorphism onto $C(X, T)$. Denote by $s$ the chain map which assigns to $c \in C(X, T)$ the element of $C(X)$ whose coordinates correspond to the successive subdivisions $S d^{i}(c)$ of $c$. If $h$ is an induced homomorphism, let $\varphi: C(X) \rightarrow C(Y)$ be $\varepsilon$-accurate and such that $\varphi_{*}=h$.

Now $s$ reduces supports, hence $\varphi s$ is the required chain map. Conversely, if $\psi: C(X, T) \rightarrow C(Y)$ is $\varepsilon / 2$-accurate and such that $\psi_{*}=h$, then $\phi p$ is the required chain map, since $p$ is $\varepsilon / 2$-accurate with respect to the identity map of $X$.

(3) Lemma. Let $X$ and $Y$ be compact polyhedra. If, given $\varepsilon>0$, there is an arbitrarily fine triangulation $T$ of $X$ and an $\varepsilon$-accurate $\psi$ : $C(X, T) \rightarrow C(Y)$ such that $\psi_{*}$ is nontrivial, then $F$ has a nontrivial induced homomorphism.

Proof. We may assume that $X$ and $Y$ are connected. Let $L$ be the finite-dimensional vector space of homomorphisms from $H(X)$ to $H(Y)$. If $\varepsilon>0$, let $A(\varepsilon)$ be the set of homomorphisms $h$ in $L$ such that $h$ preserves Kronecker index and is induced by an $\varepsilon$-accurate chain map of $C(X, T)$, where $T$ has mesh less than $\varepsilon$. By hypothesis $A(\varepsilon)$ is not empty. Furthermore one easily shows that $A(\varepsilon)$ is a coset modulo a subspace of $L$, and that if $\delta<\varepsilon$, then $A(\delta) \subset A(\varepsilon)$. Thus $\bigcap_{\varepsilon>0} A(\varepsilon)$ is not empty, but an element of this intersection is, by the preceding 
lemma, a nontrivial induced homomorphism of $F$.

$\S 2$. In order to establish the existence of nontrivial induced homomorphisms in certain cases, we need some general properties of setvalued functions. Note first that if $F: X \rightarrow Y$ is continuous and $K$ is a component of the graph $\Gamma=\{(x, y) \mid y \in F(x)\}$ of $F$, then $K$ projects onto a component of $X$. In fact, the continuity of $F$ implies that the projection $p: \Gamma \rightarrow X$ is open and closed.

(4) Lemma. Let $X$ be an arcwise connected, simply connected space, $F: X \rightarrow Y$ a set-valued map. If, for each $x \in X, F(x)$ has exactly $n$ components, then the graph $\Gamma$ of $F$ has exactly $n$ components.

Proof. Let $A=\{(x, \alpha) \mid x \in X$ and $\alpha$ is a component of $F(x)\}$. Topologize $A$ as follows. If $(x, \alpha) \in A$, select mutually disjoint neighborhoods $V_{1}, \cdots, V_{n}$ in $Y$ for the components $\alpha=\alpha_{1}, \cdots, \alpha_{n}$ of $F(x)$. Since $F$ is continuous, there is a neighborhood of $x$ such that if $x^{\prime} \in U$, then $F\left(x^{\prime}\right) \subset V_{1} \cup \cdots \cup V_{n}$ and $F\left(x^{\prime}\right)$ meets each $V_{i}$. Since $F\left(x^{\prime}\right)$ has $n$ components, there is one in each $V_{i}$. Let $U_{i}=\left\{\left(x^{\prime}, \alpha^{\prime}\right) \mid x^{\prime} \in U\right.$ and $\left.\alpha^{\prime}=F\left(x^{\prime}\right) \cup V_{i}\right\}$. The collection of all such subsets of $A$ generates a topology on $A$ for which the projection $\pi: A \rightarrow X$ is a covering map, where $\pi(x, \alpha)=x$ if $(x, \alpha) \in A$. Consequently $\pi$ is a homomorphism on each component of $A$-of which there are thus exactly $n$. If $K^{\prime}$ is a component of $A$, then $K=\left\{(x, y) \mid(x, \alpha) \in K^{\prime}\right.$ and $\left.y \in \alpha\right\}$ is a component of $\Gamma$. In fact, since $K$ is open and closed in $\Gamma$, it suffices to show that $K$ is connected, but this follows from the fact that $p \mid K$ is strongly continuous and that $p^{-1}(x) \cap K=\alpha$ is connected for each $x \in X$.

Replacing the last step above by an application of the Vietoris mapping theorem [1] we obtain :

(5) Lemma. Let $F$ be a set-valued map of a simplex $\sigma$ into an arbitrary (compact metric) space. If, for each $x \in \sigma, F(x)$ consists of exactly $n$ homologically trivial components, then the graph $\Gamma$ of $F$ consists of exactly $n$ homologically trivial components.

(6) Theorem. Let $n$ be an integer, $F: X \rightarrow Y$ a set-valued map of compact polyhedra such that if $x \in X$ then $F(x)$ is either homologically trivial or consists of $n$ homologically trivial components. Then $F$ has a nontrivial induced homomorphism.

Proof. Let $I$ be the (closed) set of points for which $F(x)$ is homologically trivial. Replace $F$ by the associated map $F^{\prime}: X \rightarrow X \times Y$. Since $X \times Y$ is a compact polyhedron and $H$ is (weakly) continuous [3], if $x \in X$ and $\varepsilon>0$ there is a $\delta_{x}$ such that the inclusion map $\delta\left(F^{\prime}(x)\right) \subset$ 
$\varepsilon\left(F^{\prime}(x)\right)$ induces the zero homology homomorphism in positive dimensions. Using the compactness of $I$ and the continuity of $F$ one can show that a $\delta$ may be selected which is independent of $x$. Thus, if the dimension of $X$ is $d \geqq 1$, there exist numbers $0<\delta<\varepsilon_{1}<\cdots \varepsilon_{d}=\varepsilon$ such that if $x \in X$ and $1 \leqq i \leqq d$ then :

(1) $\varepsilon_{i}+\varepsilon_{1}<\varepsilon_{i+1}$,

(2) each positive-dimensional cycle of $\left(\varepsilon_{i}+\varepsilon_{1}\right)\left(F^{\prime}(x)\right)$ bounds in $\varepsilon_{i+1}\left(F^{\prime \prime}(x)\right)$, and

(3) $F^{\prime}(\delta(x)) \subset \varepsilon_{1}\left(F^{\prime}(x)\right)$. Let $T$ be a triangulation of $X$ with mesh at most $\delta$, and for each simplex $\sigma$ of $T$ which meets $l$ select a point $x_{\sigma} \in \sigma \cap I$. By Lemma 3 it suffices to find an $\varepsilon$-accurate chain map $\varphi$ : $C(X, T) \rightarrow C(X \times Y)$ such that $\varphi_{*}$ is nontrivial. We may associate with each point $p$ of $X \times Y$ a 0 -chain, $\bar{p} \in C(X \times Y)$ with support $\{p\}$ and such that $\bar{p} \sim 0$ and $\bar{p} \sim \bar{p}^{\prime}$ if and only if $p$ and $p^{\prime}$ are in the same component of $X \times Y$. Define a homomorphism $\varphi_{0}: C(X, T) \rightarrow C(X \times Y)$ as follows. If the vertex $v$ is in $I$, let $\varphi_{0}(v)=n \bar{p}$, where $p \in F^{\prime}(v)$. Otherwise, let $\varphi_{0}(v)=\bar{p}_{1}+\cdots+\bar{p}_{n}$, where there is one point $p_{i}$ in each component of $F^{\prime}(v)$. Now $\varphi_{0}$ is extendable to a chain map $\varphi_{1}$ accurate with respect to $F^{\prime}$ provided that if $v w$ is a 1-simplex of $T$ then $\varphi_{0}(v) \sim \varphi_{0}(w)$ in $F^{\prime}(v w)$. Using Lemma 4 and the preceding remark, one checks this homology in case $v w$ does, or does not, meet $I$. Clearly $\varphi_{1}$ may be accurately extended to those chains in $C(X, T)$ whose supports avoid $I$. We complete the definition of $\varphi$ by an induction on dimension, defining the chain map $\varphi_{q}$ on chains of dimension at most $q$ so that $\left|\varphi_{q}(c)\right| \subset$ $\subset \varepsilon_{q}\left(F^{\prime}(|c|)\right.$. The homomorphism $\varphi_{1}$ is correctly defined. If $\varphi_{q-1}$ has been defined $(q \geqq 1)$, it suffices to define $\varphi_{q}(\sigma)$, where $\sigma$ is an oriented $q$-simplex of $T$ which meets $I$. If $\tau$ is an oriented $(q-1)$-face of $\sigma$, then

$$
\varphi_{q-1}(\tau) \subset \varepsilon_{q-1}\left(F^{\prime}(\tau)\right) \subset \varepsilon_{q-1}\left(F^{\prime}\left(\partial\left(x_{\sigma}\right)\right)\right) \subset\left(\varepsilon_{q-1}+\varepsilon_{1}\right)\left(F^{\prime \prime}\left(x_{\sigma}\right)\right) .
$$

Thus $\varphi_{q-1}(\partial \sigma)$ bounds in $\varepsilon_{q}\left(F^{\prime}(\sigma)\right)$ and $\varphi_{q}(\sigma)$ may be correctly defined.

(7) THEOREM. Let $X$ be a compact 1-dimensional polyhedron with first Betti number $R_{1} \leqq 1$. Then any set-valued map $F$ of $X$ into a compact polyhedron has a nontrivial induced homomorphism.

Proof. Given $\varepsilon>0$ and a triangulation $T$ of $X$ we must find a chain map $\varepsilon$-accurate with respect to $F^{\prime}$ and such that $\varphi_{*}$ is nontrivial. If $v w$ is a 1-simplex of $T$ and $\varphi$ is defined accurately on $v$, then $\varphi$ can be accurately defined on $w$ and $v w$ so that $\partial(\varphi(v w)=\varphi(w)-\varphi(v)$, because each component of $F(v)$ is contained in a component of $F(v w)$ which meets $F(w)$. Thus if $R_{1}=0$, the required chain map exists. If $R_{1}=1$, then $X$ may be expressed as the union of a circle $C$ and a finite num- 
ber of trees, each meeting $C$ in at most one point. If $v^{0}, v^{1}, \cdots, v^{n}$ are the vertices of $C$, we may suppose that the 1-simplexes of $C$ are $v^{0} v^{1}, v^{1} v^{2}, \cdots, v^{n} v^{0}$. Pick $y \in F\left(v^{0}\right)$ and let $\varphi^{0}\left(v^{0}\right)=\bar{y}$. There is a point $z \in F\left(v^{1}\right)$ such that if $\varphi^{0}\left(v^{1}\right)=\bar{z}$, then $\varphi^{0}\left(v^{0} v^{1}\right)$ can be correctly defined. Repeating this step we reach $\varphi^{0}\left(v^{n}\right)$, then $\varphi^{1}\left(v^{0}\right)$, and so on. Since $Y$ is a compact polyhedron there exist integers $j<k$ such that $\varphi^{k}\left(v^{0}\right)-\varphi^{j}\left(v^{0}\right)=\partial c$, with $|c| \subset \varepsilon\left(F\left(v^{0}\right)\right)$. Then on $C$ let $\varphi=\sum_{j}^{k} \varphi^{i}$, except that

$$
\varphi\left(v^{n} v^{0}\right)=\sum_{j}^{k} \varphi^{i}\left(v^{n} v^{0}\right)+c .
$$

So $\operatorname{far} \varphi$ is $\varepsilon$-accurate and accurate on vertices. But then as in the case $R_{1}=0$ this homomorphism may be extended correctly to $C(X, T)$.

We shall see in the next section that this theorem does not hold if the condition on either the dimension or the first Betti number is omitted.

§3. The Lefschetz theorem holds for set-valued functions in this form :

(8) Lemma. Let $X$ be a compact polyhedron, $F: X \rightarrow X$ an upper semi-continuous set-valued function. If $h$ is an induced homology homomorphism of $F$ and the Lefschetz number $\Lambda(h)=\sum(-1)^{q}$ trace $h_{q}$ is not zero, then $F$ has a fixed point.

The usefulness of this fact, of course, depends on our knowledge of the induced homomorphisms of a given set-valued map. From $\S 2$ we get :

(9) Theorem. Let $F$ be a set-valued self-map of a compact polyhedren $X$ such that if $x \in X, F(x)$ is homologically trivial or consists of $n$ homologically trivial components. Then $F$ has a nontrivial homomorphism $h$ such that if $\Lambda(h) \neq 0$, then $F$ has a fixed point. If, further, $X$ is homologically trivial, then $F$ has a fixed point.

The case $n=1$ is the polyhedral form of the Eilenberg-Montgomery theorem [2], except that the requirement that $F$ be lower semi-continuous is then superfluous. However, if $n>1$ upper semi-continuity alone is not sufficient. For example, consider the self-map $F$ of the Euclidean interval $[-1,1]$ for which $F(0)=\{-1,1\}$ and $F(x)$ is 1 for $x>0,-1$ for $x<0$. Also, if $n>1$ the space of induced homomorphisms need not be 1 -dimensional as in the case $n=1$.

It does not appear that this result can be generalized by altering the number of components $F(x)$ is permitted to have. Mr. Richard Dunn has shown by a series of examples (unpublished) that if $S$ is any finite set of positive integers-except certain sets of the form $\{2, n\}$ and, necessarily, $\{1, n\}$-there is a self-map $F$ of the 2-cell without fixed points 
and such that for each point $x$ the number of points in $F(x)$ occurs in $S$.

(10) THEOREM. Let $X$ be a compact 1-dimensional polyhedron with first Betti number $R_{1} \leqq 1$. Every set-valued self-map $F$ of $X$ has a nontrivial induced homomorphism such that if $\Lambda(h) \neq 0$ then $F$ has a fixed point.

In particular, as is known, every set-valued self-map of a tree has a fixed point.

The last remark of $\S 2$ may be justified by exhibiting suitable fixedpoint-free maps. As for the restriction on the Betti number for example, let $X$ be a compact connected 1-dimensional polyhedron without end points and such that $R_{1}>1$. If $\varepsilon>0$ is sufficiently small, the function $F: X \rightarrow X$ for which $F(x)=\left\{x^{\prime} \mid d\left(x, x^{\prime}\right)=\varepsilon\right\}$ will be continuous if $d$ is a suitable metric and any induced homomorphism of $F$ will be a scalar multiple of the identity homomorphism of $H(X)$. Thus a nontrivial induced homomorphism of such a function would have nonzero Lefschetz number, contradicting (8).

\section{REFERENCES}

1. E. G. Begle, The Vietoris mapping theorem for bi-compact spaces, Ann. of Math. $\mathbf{5 1}$ (1950), 534-543.

2. S. Eilenberg and D. Montgomery, Fixed point theorems for multivalued transformations, Amer. J. Math. 68 (1946), 214-222.

3. S. Eilenberg and N. Steenrod, Foundations of algebraic topology, Princeton, 1952.

4. S. Lefschetz, Algebraic topology, New York, 1942.

University of California, Los ANGeles 